\title{
Depletion of WRN enhances DNA damage in HeLa cells exposed to the benzene metabolite, hydroquinone
}

\author{
Noé Galván, Sophia Lim, Stephan Zmugg, Martyn T. Smith, Luoping Zhang* \\ Molecular Epidemiology and Toxicology Laboratory, School of Public Health, \\ University of California at Berkeley, Berkeley, CA 94720, United States
}

Received 8 June 2007; received in revised form 30 July 2007; accepted 31 July 2007

Available online 7 August 2007

\begin{abstract}
Werner syndrome is a progeroid disorder caused by mutations of the $W R N$ gene. The encoded WRN protein belongs to the family of RecQ helicases that plays a role in the maintenance of genomic stability. Single nucleotide polymorphisms in $W R N$ have been associated with an increased risk for some cancers and were recently linked to benzene hematotoxicity. To further address the role of WRN in benzene toxicity, we employed RNA interference (RNAi) to silence endogenous WRN in HeLa cells and examined the susceptibility of these WRN-depleted cells to the toxic effects of the benzene metabolite hydroquinone. HeLa cells were used as the experimental model because RNAi is highly effective in this system producing almost complete depletion of the target protein. Depletion of WRN led to a decrease in cell proliferation and an enhanced susceptibility to hydroquinone cytotoxicity as revealed by an increase in necrosis. WRN-depleted HeLa cells treated with hydroquinone also displayed an increase in the amount of DNA double-strand breaks as determined by the Comet assay, and an elevated DNA damage response as indicated by the sevenfold induction of $\gamma \mathrm{H} 2 \mathrm{AX}$ and acetyl-p53 (Lys373 and Lys382) over control levels. Together, these results show that WRN plays an important role in the protection of HeLa cells against the toxicity of the benzene metabolite hydroquinone, specifically in mounting a normal DNA damage response following the induction of DNA double-strand breaks. Further studies in bone marrow-derived stem or progenitor cells are required to confirm our findings in HeLa cells and expand our ability to extrapolate the results to benzene toxicity in humans.
\end{abstract}

(C) 2007 Elsevier B.V. All rights reserved.

Keywords: RNA interference; siRNA; $\gamma \mathrm{H} 2 \mathrm{AX}$; p53; Leukemia; Lymphoma; DNA repair; Strand breaks

\section{Introduction}

Werner syndrome is an autosomal recessive premature aging disorder characterized by the early onset of age-associated diseases, which include atherosclerosis,

Abbreviations: DSB, double-strand break; RNAi,

RNA interference; siRNA, small interfering RNA

* Corresponding author at: School of Public Health, UC Berkeley, 140 Warren Hall \#7360, Berkeley, CA 94720, United States. Tel.: +1 510643 5189; fax: +1 5106420427 .

E-mail address: luoping @ berkeley.edu (L. Zhang). osteoporosis, cataracts, and cancer [1,2]. WRN, the gene defective in Werner syndrome, encodes a member of the RecQ subfamily and the DEAH (Asp-Glu-Ala-His) subfamily of DNA and RNA helicases. It possesses an intrinsic $3^{\prime}-5^{\prime}$ DNA helicase activity as well as $3^{\prime}-5^{\prime}$ exonuclease activity. Furthermore, WRN plays important roles in the monitoring of genomic integrity and the control of cellular responses to genotoxic stress [1]. More specifically, WRN helps to recruit the proper DNA repair factors to the site of DNA strand breaks and directly interacts with p53 to modulate cell death. In fibroblast cell lines, lack of WRN results in the deregulation of DNA 
damage monitoring and the activation of DNA repair or apoptosis in response to certain types of DNA damage [3]. WRN also plays a key role in DNA double-strand break (DSB) repair [4].

Single nucleotide polymorphisms (SNPs) in WRN have been associated with an increased risk of some cancers, including non-Hodgkin lymphoma [5] and familial breast cancer [6]. In collaboration with colleagues from the National Cancer Institute and China CDC, we recently reported that four SNPs located in the functional domains of WRN (Ex4-16 G $>$ A, Ex6 $+9 \mathrm{C}>\mathrm{T}$, Ex20 - 88 G $>\mathrm{T}$ and Ex26 - 12 T>G) were associated with benzene hematotoxicity [7]. Exposure to benzene caused a decrease in total white blood cells, granulocytes and lymphocytes even among workers with relatively low levels of exposure to benzene [8]. These hematotoxic effects are thought to be caused by the metabolism of benzene to its reactive oxygen speciesgenerating metabolites, the principal metabolite being hydroquinone [9]. Hydroquinone is known to directly and indirectly cause DNA DSBs, one of the most severe lesions within the genome [10]. Additionally, hydroquinone is able to induce chromosomal instability and may contribute to the development of leukemia through this mechanism [11]. Thus, proteins such as WRN, which play a role in DSB repair and the prevention of genomic instability, would be expected to play a role in the resistance to benzene toxicity in many cell types.

In order to further examine the relationship between WRN and the cytotoxicity of hydroquinone, we used RNA interference (RNAi) to deplete endogenous WRN to determine its effect on hydroquinone-induced toxicity in vitro. In the present study, WRN was silenced in HeLa cells using siRNA, the functional unit in RNAi, and examined the resultant cytotoxicity and genotoxicity of hydroquinone by measuring its effects on cell proliferation, apoptosis or necrosis, DNA strand breaks, and the induction of DNA damage response proteins. More specifically, we measured the levels of $\gamma \mathrm{H} 2 \mathrm{AX}$ and acetyl-p53 (Lys373 and Lys382), translationally post-modified proteins associated with the DNA damage response [12-14] to assess the DNA damage response. HeLa cells were used as the experimental model because RNAi is highly effective in this system producing almost complete depletion of the target protein.

\section{Materials and methods}

\subsection{Cell culture and hydroquinone treatment}

HeLa cells, obtained from the American Type Culture Collection (Manassas, VA), were used as the experimental model for this study due to the ease of transfection with siRNA and the ability to produce $>90 \%$ knockdown of specific mRNA expression. HeLa cells were maintained in Dulbecco's modified Eagle's medium (DMEM) (Invitrogen, Carlsbad, CA) containing 10\% FBS (Omega Scientific, Tarzana, CA) and antibiotic solution $(100 \mu \mathrm{g} / \mathrm{mL}$ penicillin and $100 \mathrm{IU} / \mathrm{mL}$ streptomycin) at $37^{\circ} \mathrm{C}$ in a $5 \% \mathrm{CO}_{2}$ incubator.

Hydroquinone (CAS Registry No. 123-31-9) was purchased from Sigma Chemical (St. Louis, MO) and dissolved in $1 \times$ PBS for treatment of cells. In order to ascertain the appropriate non-cytotoxic dose of hydroquinone, a dose-response curve (0-400 $\mu \mathrm{M}$ hydroquinone) was determined in non-transfected HeLa cells using the trypan blue exclusion assay. After $48 \mathrm{~h}$ of exposure, the cytotoxic doses for hydroquinone-treated HeLa cells were determined to be those greater than $150 \mu \mathrm{M}$. As such, cell proliferation assays were conducted at $100 \mu \mathrm{M}$ hydroquinone while all other assays were done at $150 \mu \mathrm{M}$, where maximal effective responses could be detected.

\section{2. siRNA and transfection}

Specific target siRNA duplexes were designed using Invitrogen's BLOCK-iT ${ }^{\mathrm{TM}}$ RNAi Designer (Carlsbad, CA), which employs a rigorous algorithm to control for and prevent non-specific knock down. After testing several siRNA constructs for their knock down efficiency, the following siRNA sequences were determined and used in the experiments for the human WRN mRNA (NM_00053), 5'-CCGAGGAUCUAAUUCUCAGCGUCUU$3^{\prime}$ and for non-specific scrambled (NS) siRNA, 5'-CCGUAGAUCUUACUCCGAUGAGCUU- $3^{\prime}$. Asynchronous HeLa cells were seeded at $0.3 \times 10^{6}$ cells/well in six-well tissue culture plates and allowed to reach $\sim 60 \%$ confluency within $24 \mathrm{~h}$. HeLa cells were transfected with either NS or WRN siRNA $(5 \mathrm{nM})$ using Lipofectamine ${ }^{\mathrm{TM}} 2000$ (Invitrogen) according to the manufacturer's instructions. The transfection efficiency of $>95 \%$ was determined by fluorescence microscopy using BLOCK-iT ${ }^{\mathrm{TM}}$ Fluorescent Oligo labeled with FITC (Invitrogen).

\subsection{Cell proliferation and apoptosis analysis}

Following gene silencing as described above, the HeLa cells were treated with $100 \mu \mathrm{M}$ hydroquinone for $0,1,2$, and 3 days. The hydroquinone treated cells were then washed twice with $1 \times$ PBS, collected by trypsinization, and combined with complete media. Cells were enumerated using a hemocytometer to determine cell proliferation; the trypan blue exclusion assay was also utilized to assess cell viability. Cell proliferation was measured in two independent experiments.

Flow cytometric analysis was used to determine apoptosis and necrosis in HeLa cells treated with $150 \mu \mathrm{M}$ hydroquinone for $48 \mathrm{~h}$ following WRN silencing. This exposure time was determined to allow for hydroquinone to induce apoptosis and necrosis. Cells were collected with $1 \times$ PBS, trypsinized and stained with propidium iodide (PI) and Annexin V-FITC 
according to the manufacturer's protocol (BD Pharmingen, San Diego, CA). In three independent experiments, at least $10^{4}$ cells were analyzed on a Beckman Coulter EPICS XLMCL flow cytometer using System II software for each experiment.

\subsection{Single-cell gel electrophoresis (Comet)}

The alkaline Comet assay was performed as previously described with some modifications [15]. Cells were exposed to $150 \mu \mathrm{M}$ hydroquinone for 6,12 and $24 \mathrm{~h}$ prior to preparation for Comet analysis of DNA damage. Five hundred randomly chosen cells per slide were scanned and analyzed automatically using CometScan imaging software (MetaSystems, Germany). Cells were subsequently screened manually to exclude cells that did not meet stringent requirements (i.e. poor staining, out of focus, or oddly shaped). All slides were coded to prevent observer bias. Data is presented for the tail moments resulting at the $12 \mathrm{~h}$ time point because the maximum response was detected at this time. Tail moment combines both measures of tail length and tail intensity in the Comet assay.

\subsection{Western blot analysis}

Following siRNA transfection, HeLa cells were treated with $150 \mu \mathrm{M}$ hydroquinone for $24 \mathrm{~h}$, which was the optimal observed time to allow for the expression of the translationally modified proteins. Total cell lysates were then collected from six-well tissue plates using $200 \mu \mathrm{L}$ radioimmunoprecipitation assay (RIPA) lysis buffer per well $(50 \mathrm{mM}$ Tris-HCl-pH 7.4, $150 \mathrm{mM} \mathrm{NaCl}, 1 \mathrm{mM}$ EDTA, 1 mM EGTA, $1 \mathrm{mM} \mathrm{Na} \mathrm{VO}_{4}, 1 \%$ NP40, $0.25 \%$ DOC, $0.1 \%$ SDS, $1 \mathrm{mM}$ PMSF, $1 \mu \mathrm{g} / \mathrm{mL}$ Leupeptin, $1 \mu \mathrm{g} / \mathrm{mL}$ Aprotinin). Protein concentrations were determined by the Bradford assay (Bio-Rad, Hercules, CA). Equal protein concentrations were resolved in a $7.5 \%$ polyacrylamide gel using sodium dodecyl sulfatepolyacrylamide gel electrophoresis (SDS-PAGE), transferred onto a nitrocellulose membrane, and immunoblotted for WRN, p53, Ac-p53, $\gamma \mathrm{H} 2 \mathrm{AX}$ and actin. Antibodies anti-acetyl-p53 (Lys373 and Lys382) and anti- $\gamma \mathrm{H} 2 \mathrm{AX}$ were purchased from Upstate Biotechnology (Charlottesville, VA); anti-actin (I-19), anti-WRN (H-300) and anti-p53 (DO-1) were obtained from Santa Cruz Biotechnology (Santa Cruz, CA). Protein was visualized using the enhanced chemiluminescent (ECL) method according to the manufacturer's protocol (Amersham Biosciences, UK). Film was exposed and developed using the Konica SRX-101 developer (Konica Minolta Medical Imaging USA, Wayne, NJ). Band intensities were subsequently quantified using Imagequant software.

\subsection{Statistical analysis}

For cell proliferation, linear regression analysis was used to compare the trend differences among treatment groups. All other statistical analyses were performed by one-way analysis of variance (ANOVA) followed by Tukey multiple comparison tests for multiple groups.

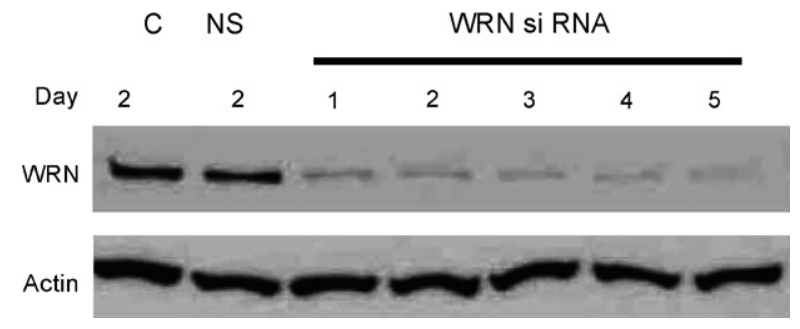

Fig. 1. Depletion of WRN in HeLa cells by RNA interference. HeLa cells remained untransfected (C) or were transfected with $5 \mathrm{nM}$ of either non-specific siRNA (NS) or WRN-specific siRNA (WRN). Whole protein lysates were collected for up to 5 days after transfection and subjected to western blot analysis as described in Section 2. WRN knockdown levels were determined by normalizing against levels of actin, the loading control. WRN expression was depleted by more than $90 \%$ for up to 5 days.

\section{Results}

\subsection{WRN depletion by RNA interference}

The effect of control (NS) and WRN siRNA on WRN protein expression in HeLa cells was first examined. HeLa cells were transfected at $\sim 60 \%$ confluency with $5 \mathrm{nM}$ siRNA and protein levels were subsequently assayed by western blot analysis over the course of 5 days. The suppression of endogenous WRN expression in siRNA-transfected cells was achieved at levels of $81 \%$ within $24 \mathrm{~h}$ and $90 \%$ by $48 \mathrm{~h}$ (Fig. 1). WRN levels remained suppressed at levels greater than $90 \%$ 3-5 days post-transfection (Fig. 1). In contrast, cells not transfected $(\mathrm{C})$ or those transfected with $5 \mathrm{nM}$ nonspecific scrambled (NS) siRNA, constitutively expressed WRN protein. These results demonstrate knockdown specificity and indicate that WRN siRNA effectively suppresses the expression of endogenous WRN.

\subsection{Cell proliferation following WRN depletion and hydroquinone treatment}

Following a $48 \mathrm{~h}$ transfection with siRNA, HeLa cells were exposed to $100 \mu \mathrm{M}$ hydroquinone for $0,1,2$, and 3 days. At each time point, cells were enumerated and plotted against treatment day (0 day) to determine the resultant proliferation capacities (Fig. 2). In the unexposed cell cultures (NT) transfected with scrambled non-specific siRNA (NS), the number of cells more than doubled over the 3 days. Treatment with either hydroquinone or WRN specific siRNA resulted in a decrease of proliferation capacity $(\sim 50 \%)$ approximately equally and significantly $(p<0.01)$, but the two in combination produced an almost complete suppression of growth $(\sim 14 \%)$ that was highly significant ( $p<0.0001$, Fig. 2$)$. 


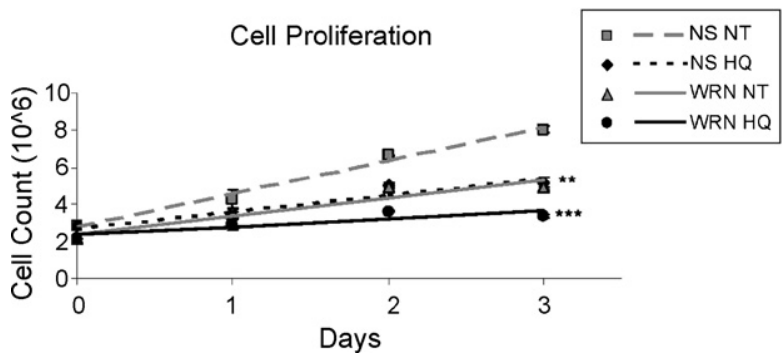

Fig. 2. Decreased proliferation of HeLa cells following WRN depletion and hydroquinone treatment. Non-specific siRNA (NS) or WRN-specific siRNA (WRN) pretreated HeLa cells were exposed to $100 \mu \mathrm{M}$ Hydroquinone (HQ) for 0, 1, 2, and 3 days. Cell proliferation was evaluated using a hemocytometer with the trypan blue exclusion assay in unexposed cultures (NT), and in those treated with hydroquinone (HQ). Treatment with either HQ or WRN-specific siRNA reduced proliferation rates by approximately $50 \%(* * p<0.01)$. When HQ and WRN siRNA were used in combination, proliferation was inhibited to $14 \%$ of the control $(* * * p<0.001)$. The data represents the average of two independent experiments.

\subsection{Apoptosis and necrosis in WRN-depleted HeLa cells following hydroquinone treatment}

Flow cytometric analysis was used to determine the percentage of apoptotic and necrotic cells in cell cultures treated with and without $150 \mu \mathrm{M}$ hydroquinone for $48 \mathrm{~h}$. As the detection of early apoptosis is a time sensitive endpoint, apoptosis levels were observed at several time points and the $48 \mathrm{~h}$ time point was determined to be optimal for the detection of hydroquinone-induced apoptosis/necrosis in WRN-deficient cells. In the control cultures incubated with non-specific (NS) siRNA, hydroquinone treatment produced little change in the percentage of apoptotic cells, but caused a significant 1.7-fold increase in the percentage of necrotic cells $(p<0.05$, Fig. 3A). Depletion of WRN with specific siRNA had no significant effect on the percentage of apoptotic or necrotic cells after $48 \mathrm{~h}$. Treatment of the WRN-depleted cultures with hydroquinone however caused a highly significant 2.5 -fold increase in the percentage of necrotic cells in comparison with the percentage found in WRN-depleted unexposed controls $(p<0.001$, Fig. 3A). Furthermore, the percentage of necrotic cells after hydroquinone treatment in WRNdepleted cells was significantly greater than in NS control cultures $(p<0.01)$. No significant effects were observed on the percentage of apoptotic cells in these cultures.

When intact cells were examined alone by flow cytometry, hydroquinone's effects were even more striking (Fig. 3B). An approximately threefold increase $(p<0.001)$ was seen in the necrotic population of the
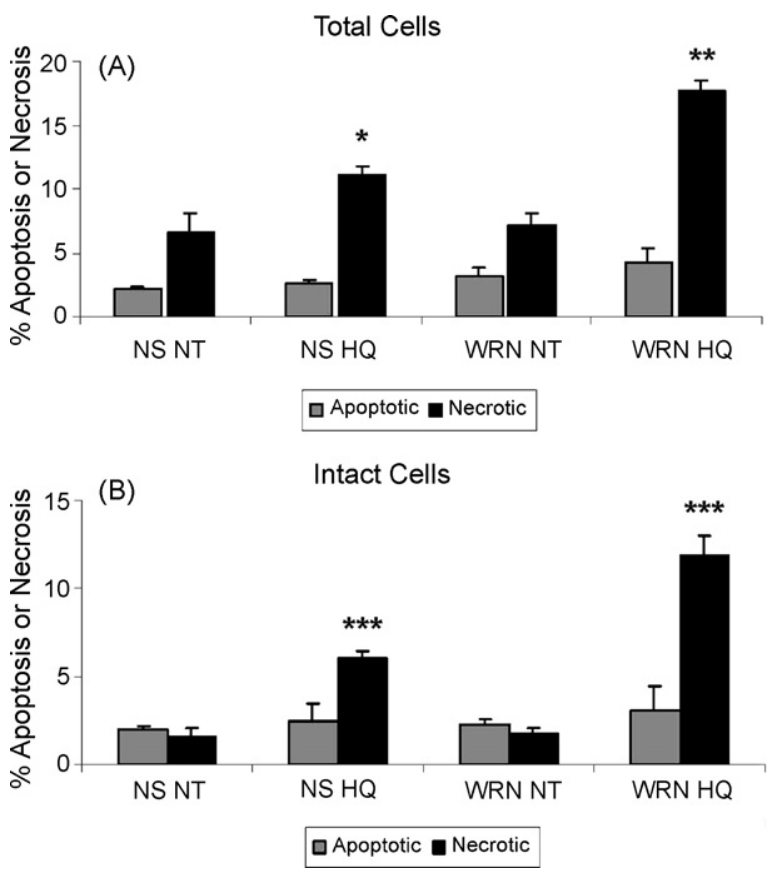

Fig. 3. Flow cytometric analysis of apoptosis and necrosis in HeLa cells following WRN depletion and hydroquinone treatment. HeLa cells treated with $150 \mu \mathrm{M}$ hydroquinone (HQ) for $48 \mathrm{~h}$ were stained with Annexin V and propidium iodide (PI), and subjected to flow cytometric analysis. (A) A gate parameter to include total cells was set to analyze apoptosis (gray bar, positive for Annexin $\mathrm{V}$ and negative for PI) and necrosis (black bar, positive for both Annexin $\mathrm{V}$ and PI) before and after HQ treatment. * $p<0.05$ vs. NS NT; ** $p<0.01$ vs. WRN NT and NS HQ. (B) A gate parameter to exclude cell debris was set to analyze intact cells for apoptosis and necrosis before and after HQ treatment. $* * * p<0.001$ vs. NS NT, NS HQ and WRN NT. The mean \pm S.D. of three independent experiments is presented.

cells pretreated with non-specific (NS) siRNA following hydroquinone treatment. However, a significantly greater than sevenfold increase $(p<0.001)$ was observed after the hydroquinone treatment of WRN-depleted cells as compared to their unexposed controls. Additionally, the percentage of necrotic cells after hydroquinone treatment in WRN-depleted cells was significantly greater than in undepleted NS control cultures $(p<0.001)$. No significant effects were observed in the percentage of apoptotic cells. These results demonstrate that hydroquinone $(150 \mu \mathrm{M})$ produces higher levels of necrosis in WRN-depleted cells than in undepleted WRN cells.

\subsection{DNA damage in WRN-depleted HeLa cells following hydroquinone treatment}

siRNA treated HeLa cells were either exposed to $150 \mu \mathrm{M}$ hydroquinone or remained unexposed (NT) for $12 \mathrm{~h}$ and the ensuing DNA damage was evaluated using 


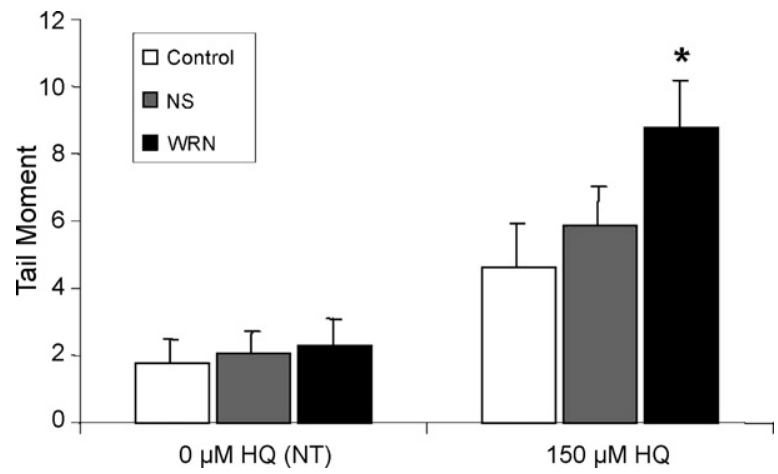

Fig. 4. WRN depletion of HeLa cells increases susceptibility to hydroquinone (HQ)-induced DNA strand breaks. HeLa cells were treated with no HQ or $150 \mu \mathrm{M}$ HQ for $12 \mathrm{~h}$ and DNA strand breaks were evaluated by the single-cell gel electrophoresis assay (Comet). DNA tail moment was analyzed in untreated cultures (NT) and cultures treated with HQ, following pretreatment with control (white bar), non-specific siRNA (NS, gray bar), and WRN-specific siRNA (WRN, black bar). ${ }^{*} p<0.05$ vs. control and NS. The mean \pm S.D. of three independent experiments is presented.

the single-cell gel electrophoresis (Comet) assay, which detects DNA strand breaks. DNA damage repair by proteins such as WRN ensues quite rapidly within the cell. As such, to effectively detect any DNA damage in WRNdepleted cells, it was necessary to obtain a time point in which hydroquinone related DNA repair had not yet occurred. While earlier and later time points were taken into account, the $12 \mathrm{~h}$ time point demonstrated the maximum detectable DNA damage within all cell culture types. In the unexposed cell cultures (NT), there were no significant differences in the amount of DNA strand breaks as measured by the Comet tail moment between cell cultures pretreated with siRNA, non-specific scrambled siRNA (NS) or WRN-specific siRNA (WRN), and those receiving no pretreatment with siRNA (control) (Fig. 4). A significant increase in tail moment was seen in all three types of cell culture following treatment with $150 \mu \mathrm{M}$ hydroquinone $(p<0.001)$ (Fig. 4). However, DNA damage from hydroquinone treatment was significantly greater in the WRN-depleted cultures pretreated with WRN-specific siRNA (WRN) than in the control $(p<0.001)$ and NS $(p<0.05)$ hydroquinone-treated cell cultures (Fig. 4). These results provide additional evidence that WRN-depletion enhances the genotoxicity of hydroquinone.

\subsection{Effect of hydroquinone treatment and WRN-depletion on the DNA damage response}

Following the induction of DNA damage, cells mount a response that involves p53 and the localization of

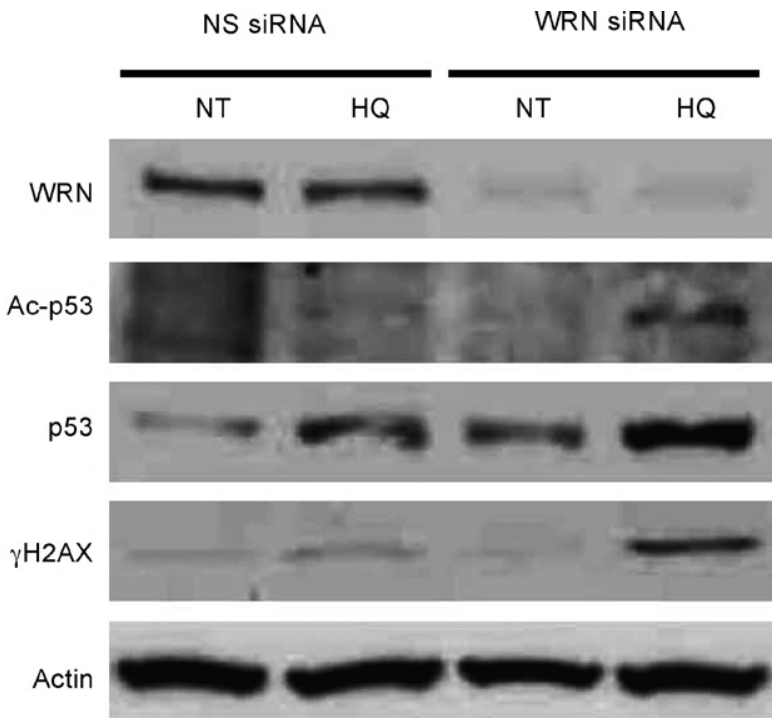

Fig. 5. Increase in DNA damage response following WRN depletion and hydroquinone treatment. Following $150 \mu \mathrm{M}$ hydroquinone (HQ) treatment or no treatment (NT) for $24 \mathrm{~h}$, total protein was extracted and immunoblotted for WRN, $\gamma \mathrm{H} 2 \mathrm{AX}, \mathrm{p} 53$ and acetylated-p53 (Lys373 and Lys382). Expression levels were normalized against the loading control actin. Non-specific siRNA (NS) pretreated HeLa cells maintained constitutive levels of WRN expression; WRN-specific siRNA (WRN) pretreated HeLa cells resulted in $>90 \%$ knockdown. The immunoblot is representative of two independent experiments.

$\gamma \mathrm{H} 2 \mathrm{AX}$ to DNA double-strand breaks $[12,14] . \mathrm{p} 53$ is stabilized and activated in response to cellular stress through post-translational modifications which include acetylation [16]. Acetylation of p53 at Lys373 and Lys382 increases the protein's stability, which in turn influences its interactions with other proteins (MDM2 and histone deacetylases) and alters the expression of genes that can affect cell cycle arrest and apoptosis $[17,18]$. Immunoblot analysis was used to determine levels of p53, acetylated p53 (Lys373 and Lys382), and $\gamma \mathrm{H} 2 \mathrm{AX}$ following WRN-depletion by siRNA and $24 \mathrm{~h}$ hydroquinone treatment (Fig. 5). Hydroquinone treatment $(150 \mu \mathrm{M})$ alone caused a greater than fivefold increase in p53 protein levels, but only limited effects, if any, on the levels of acetylated p53 and $\gamma \mathrm{H} 2 \mathrm{AX}$ (compare first and second column in Fig. 5). Likewise, pretreatment of cells with WRN-specific siRNA also caused elevated levels of p53, but no change in the expression levels of acetylated p53 or $\gamma \mathrm{H} 2 \mathrm{AX}$ when compared to cells pretreated with non-specific scrambled siRNA (compare first and third columns in Fig. 5). However, the DNA damage response to hydroquinone treatment was dramatically enhanced in WRN-depleted cells as seen by the induction of p53, acetylated p53 and $\gamma \mathrm{H} 2 \mathrm{AX}$ levels (compare third and 
fourth columns in Fig. 5). The enhanced response was especially pronounced in acetylated p53 and $\gamma \mathrm{H} 2 \mathrm{AX}$, where a synergism between WRN depletion and hydroquinone treatment was observed to lead to sevenfold increases in expression over control levels.

\section{Discussion}

Genetic association studies have implicated WRN as playing an important role in the susceptibility to benzene toxicity and risk of non-Hodgkin lymphoma [5,7]. WRN organizes the response to DNA damage and is critical to the repair of DNA double-strand breaks (DSBs). DSBs are highly toxic lesions that, if not repaired or repaired incorrectly, can cause cell death, mutations, and chromosomal translocations that can lead to cancer $[19,20]$.

In the present study we further explored the role of WRN in susceptibility to the cytotoxic effects from the benzene metabolite hydroquinone using RNA interference. We employed siRNA specific to WRN, which readily depleted WRN protein levels $>90 \%$ in HeLa cells. As we are addressing protein function, HeLa cells were chosen as the experimental model for this study due to the ease of transfection and the susceptibility to longterm knockdown using siRNA. It is difficult to achieve this same level of knockdown in suspension cells such as leukemic cell lines because of technical limitations in the delivery of siRNA. WRN depletion in HeLa cells caused a decrease in cell proliferation, but no significant increases in the amount of DNA strand breaks or levels of apoptosis or necrosis were detected. The observed decrease in cell proliferation is in agreement with previous reports, which showed that the depletion of WRN decreased cell growth and increased senescence as determined by the measurement of SA- $\beta-$ Gal activity $[3,21]$.

WRN depletion greatly enhanced the cytotoxic and genotoxic effects of hydroquinone. In WRN-depleted cells, hydroquinone treatment caused an almost total inhibition of cell proliferation at the non-cytotoxic dose and increased the levels of both DNA strand breaks and necrosis such that they were significantly above that seen in the control cultures pretreated with scrambled non-specific siRNA. Expression of p53 was also slightly elevated in the WRN-depleted cells, but there was no significant change in the expression of $\gamma \mathrm{H} 2 \mathrm{AX}$ or acetylated p53. Only after the WRN-depleted HeLa cells were exposed to hydroquinone, effects were seen. There was an additive induction of p53 levels and synergistic inductions of acetylated p53 (Lys373 and Lys382) and $\gamma \mathrm{H} 2 \mathrm{AX}$, as seen by the sevenfold increases in expression levels of these post-translationally modified proteins over that of their respective control levels.
WRN is also known to associate with $\gamma \mathrm{H} 2 \mathrm{AX}$ via Nbs1, a member of the M/R/N complex, during WRN's recruitment to the site of DNA DSBs [22]. The observed increase in the expression of $\gamma \mathrm{H} 2 \mathrm{AX}$ could be an attempt by the WRN-depleted cells to recruit other DNA helicase proteins with functions similar to WRN, to repair the DNA damage. However, since there is a decrease in cell proliferation and a large increase in necrosis in WRN-depleted HeLa cells treated with hydroquinone, our model suggests that the level of redundancy in DNA repair is very limited at least in HeLa cells. This observation is supported by previous work showing that there is limited redundancy among DNA helicases (WRN, BLM, $\mathrm{XPB}$ and XPD) [23]. The limited redundancy in repairing DNA damage caused by hydroquinone may slow DNA strand break repair, leading to an increase in chromosomal aberrations detrimental to genome integrity.

We found an additive induction of p53 and a synergistic induction of acetylated p53 (Lys373 and Lys382) following hydroquinone treatment of WRN-depleted HeLa cells. The increase of p53 in WRN-depleted HeLa cells was not unexpected as it is well established that WRN and p53 interact physically and functionally $[24,25]$. WRN is important in inducing an effective p53 response to DNA damage [26], suggesting that these proteins may cooperate to maintain genomic stability. Furthermore, the tumor suppressor p53 responds to a large array of stress signals to regulate processes such as senescence, cell cycle, cell death, and DNA damage. The activity of p53 is regulated by post-translational modifications, such as the phosphorylation of serine and/or threonines, and the acetylation, ubiquitylation and sumoylation of lysine residues, which in turn dictate the biological activity of p53 [16,27]. In the present study we used an antibody that recognizes the acetylation of p53 at Lys373 and Lys382. Acetylation of p53 at Lys373 induces cell cycle arrest and apoptosis, whereas acetylation of p53 at Lys382 induces only cell cycle arrest [17]. Even though our antibody does not distinguish between Lys373 and Lys382, the fact that a decrease in cell proliferation was observed without induction of apoptosis suggests that Lys382 is the preferred posttranslational modification in WRN-depleted cells treated with hydroquinone. Further work is necessary to confirm this. The observed increase in $\gamma \mathrm{H} 2 \mathrm{AX}$ and acetylated p53 suggests that HeLa cells depleted of WRN are more susceptible to DNA damage induced by hydroquinone.

Our findings further implicate the importance of DNA DSBs in hydroquinone cytotoxicity and thus, by inference, in benzene toxicity. Earlier work by Amin and Witz showed that hydroquinone induced DSBs in HL-60 cells [28]. As suggested by Winn who recently showed 
that the phenolic and quinone metabolites of benzene induce homologous recombination [10], inappropriate repair of these DSBs could lead to hyper-recombination. DNA damage from hydroquinone could therefore lead to chromosome rearrangements and genomic instability if DSB repair is inefficient. This may in turn cause direct hematotoxic effects or the initiation of leukemia or lymphoma. These conclusions are supported by the genetic association studies, which implicate DNA DSB repair as being a susceptibility factor for benzene hematotoxicity [7]. However, further studies involving the adaptation of our experimental model to hematopoietic cells are necessary to confirm our findings in HeLa cells and expand our ability to extrapolate the results to benzene toxicity in humans. Finally, our results show the utility of RNA interference in human cell culture for the further exploration of susceptibility genes previously implicated in genetic association studies.

\section{Acknowledgements}

This work was supported by NIH grant P42 ES04705 and R01 ES06721. N.G. was supported by a Research Supplement to Promote Diversity in Health-Related Research to Grant R01 ES06721 from NIEHS. The authors would like to thank Dr. Zhiying Ji for his assistance in statistical analysis and transfection of cultured cells.

\section{References}

[1] L. Comai, B. Li, The Werner syndrome protein at the crossroads of DNA repair and apoptosis, Mech. Ageing Dev. 125 (2004) 521-528.

[2] C.J. Epstein, G.M. Martin, A.L. Schultz, A.G. Motulsky, Werner's syndrome a review of its symptomatology, natural history, pathologic features, genetics and relationship to the natural aging process, Medicine (Baltimore) 45 (1966) 177-221.

[3] A.M. Szekely, F. Bleichert, A. Numann, S. Van Komen, E. Manasanch, A. Ben Nasr, A. Canaan, S.M. Weissman, Werner protein protects non-proliferating cells from oxidative DNA damage, Mol. Cell Biol. 25 (2005) 10492-10506.

[4] L. Lan, S. Nakajima, K. Komatsu, A. Nussenzweig, A. Shimamoto, J. Oshima, A. Yasui, Accumulation of Werner protein at DNA double-strand breaks in human cells, J. Cell Sci. 118 (2005) 4153-4162.

[5] M. Shen, T. Zheng, Q. Lan, Y. Zhang, S.H. Zahm, S.S. Wang, T.R. Holford, B. Leaderer, M. Yeager, R. Welch, D. Kang, P. Boyle, B. Zhang, K. Zou, Y. Zhu, S. Chanock, N. Rothman, Polymorphisms in DNA repair genes and risk of non-Hodgkin lymphoma among women in Connecticut, Hum. Genet. 119 (2006) 659-668.

[6] M. Wirtenberger, B. Frank, K. Hemminki, R. Klaes, R.K. Schmutzler, B. Wappenschmidt, A. Meindl, M. Kiechle, N. Arnold, B.H. Weber, D. Niederacher, C.R. Bartram, B. Burwinkel, Interaction of Werner and Bloom syndrome genes with p53 in familial breast cancer, Carcinogenesis 27 (2006) 1655-1660.
[7] M. Shen, Q. Lan, L. Zhang, S. Chanock, G. Li, R. Vermeulen, S.M. Rappaport, W. Guo, R.B. Hayes, M. Linet, S. Yin, M. Yeager, R. Welch, M.S. Forrest, N. Rothman, M.T. Smith, Polymorphisms in genes involved in DNA double-strand break repair pathway and susceptibility to benzene-induced hematotoxicity, Carcinogenesis 27 (2006) 2083-2089.

[8] Q. Lan, L. Zhang, G. Li, R. Vermeulen, R.S. Weinberg, M. Dosemeci, S.M. Rappaport, M. Shen, B.P. Alter, Y. Wu, W. Kopp, S. Waidyanatha, C. Rabkin, W. Guo, S. Chanock, R.B. Hayes, M. Linet, S. Kim, S. Yin, N. Rothman, M.T. Smith, Hematotoxicity in workers exposed to low levels of benzene, Science 306 (2004) 1774-1776.

[9] P. Kolachana, V.V. Subrahmanyam, K.B. Meyer, L. Zhang, M.T. Smith, Benzene and its phenolic metabolites produce oxidative DNA damage in HL60 cells in vitro and in the bone marrow in vivo, Cancer Res. 53 (1993) 1023-1026.

[10] L.M. Winn, Homologous recombination initiated by benzene metabolites: a potential role of oxidative stress, Toxicol. Sci. 72 (2003) 143-149.

[11] I.D. Gowans, S.A. Lorimore, J.M. McIlrath, E.G. Wright, Genotype-dependent induction of transmissible chromosomal instability by gamma-radiation and the benzene metabolite hydroquinone, Cancer Res. 65 (2005) 3527-3530.

[12] D.R. Pilch, O.A. Sedelnikova, C. Redon, A. Celeste, A. Nussenzweig, W.M. Bonner, Characteristics of gamma-H2AX foci at DNA double-strand breaks sites, Biochem. Cell Biol. 81 (2003) 123-129.

[13] K. Sakaguchi, J.E. Herrera, S. Saito, T. Miki, M. Bustin, A. Vassilev, C.W. Anderson, E. Appella, DNA damage activates p53 through a phosphorylation-acetylation cascade, Genes Dev. 12 (1998) 2831-2841.

[14] O. Fernandez-Capetillo, A. Lee, M. Nussenzweig, A. Nussenzweig, H2AX: the histone guardian of the genome, DNA Repair (Amst) 3 (2004) 959-967.

[15] N.P. Singh, R.E. Stephens, X-ray induced DNA doublestrand breaks in human sperm, Mutagenesis 13 (1998) $75-79$.

[16] A.M. Bode, Z. Dong, Post-translational modification of p53 in tumorigenesis, Nat. Rev. Cancer 4 (2004) 793805.

[17] S. Roy, K. Packman, R. Jeffrey, M. Tenniswood, Histone deacetylase inhibitors differentially stabilize acetylated p53 and induce cell cycle arrest or apoptosis in prostate cancer cells, Cell Death Differ. 12 (2005) 482-491.

[18] A. Ito, C.H. Lai, X. Zhao, S. Saito, M.H. Hamilton, E. Appella, T.P. Yao, p300/CBP-mediated p53 acetylation is commonly induced by $\mathrm{p} 53$-activating agents and inhibited by MDM2, Embo. J. 20 (2001) 1331-1340.

[19] S.P. Jackson, Sensing and repairing DNA double-strand breaks, Carcinogenesis 23 (2002) 687-696.

[20] J. Whysner, M.V. Reddy, P.M. Ross, M. Mohan, E.A. Lax, Genotoxicity of benzene and its metabolites, Mutat. Res. 566 (2004) 99-130.

[21] C. Grandori, K.J. Wu, P. Fernandez, C. Ngouenet, J. Grim, B.E. Clurman, M.J. Moser, J. Oshima, D.W. Russell, K. Swisshelm, S. Frank, B. Amati, R. Dalla-Favera, R.J. Monnat Jr., Werner syndrome protein limits MYC-induced cellular senescence, Genes Dev. 17 (2003) 1569-1574.

[22] W.H. Cheng, S. Sakamoto, J.T. Fox, K. Komatsu, J. Carney, V.A. Bohr, Werner syndrome protein associates with gamma H2AX in a manner that depends upon Nbs1, FEBS Lett. 579 (2005) 1350-1356. 
[23] E.A. Spillare, X.W. Wang, C. von Kobbe, V.A. Bohr, I.D. Hickson, C.C. Harris, Redundancy of DNA helicases in p53-mediated apoptosis, Oncogene 25 (2006) 2119-2123.

[24] R.M. Brosh Jr., V.A. Bohr, Roles of the Werner syndrome protein in pathways required for maintenance of genome stability, Exp. Gerontol. 37 (2002) 491-506.

[25] R.M. Brosh Jr., P. Karmakar, J.A. Sommers, Q. Yang, X.W. Wang, E.A. Spillare, C.C. Harris, V.A. Bohr, p53 Modulates the exonuclease activity of Werner syndrome protein, J. Biol. Chem. 276 (2001) 35093-35102.
[26] G. Blander, J. Kipnis, J.F. Leal, C.E. Yu, G.D. Schellenberg, M. Oren, Physical and functional interaction between p53 and the Werner's syndrome protein, J. Biol. Chem. 274 (1999) 29463-29469.

[27] C. Prives, J.L. Manley, Why is p53 acetylated? Cell 107 (2001) $815-818$.

[28] R.P. Amin, G. Witz, DNA-protein crosslink and DNA strand break formation in HL-60 cells treated with trans,trans-muconaldehyde, hydroquinone and their mixtures, Int. J. Toxicol. 20 (2001) 69-80. 